\title{
Effects of oral administration of gelatin capsules on plasma insulin and glucagon levels in dogs
}

\author{
Hideaki Yokoyama, Taku Masuyama, Tomio Ishida, Yusuke Suzuki, Toshiyuki Shoda, \\ Hideyuki Kuno and Shoichiro Sugai
}

Toxicology Research Lab., Central Pharmaceutical Research Institute, JAPAN TOBACCO INC., 23 Naganuki Hadano, Kanagawa 257-0024, Japan

(Received January 23, 2017; Accepted January 27, 2017)

\begin{abstract}
A gelatin capsule is widely used as a device for oral administration of granular/powdered materials or small amounts of liquid materials in animal experiments. In this study, we investigated the insulin- and glucagon-related parameters following oral administration of gelatin capsules to beagle dogs since a gelatin capsule is made of protein composed of many amino acids as known to stimulate insulin or glucagon secretion. Gelatin capsules (1/2 ounces in size) were administered once orally to three male beagle dogs at the dose level of 0.29 and $1.67 \mathrm{~g} / \mathrm{kg}$ as gelatin and the plasma levels of insulin, glucagon, glucose, non-esterified fatty acids (NEFA), total ketone bodies (KB) and alanine were determined before dosing and for up to $4 \mathrm{hr}$ after dosing. Plasma insulin, glucagon and alanine levels increased immediately after dosing and subsequently plasma NEFA and total KB levels decreased. Most of these changes disappeared by $4 \mathrm{hr}$ after dosing. No effects on plasma glucose levels were noted. In conclusion, a single oral administration of gelatin capsules increased plasma insulin and glucagon levels transiently and altered plasma levels of some biochemical parameters in dogs. These results might imply that attention should be paid to their own effect on the insulin and glucagon secretion at conducting animal experiments using a lot of gelatin capsules.
\end{abstract}

Key words: Amino acid, Beagle dog, Gelatin capsule, Glucagon, Insulin

\section{INTRODUCTION}

A gelatin capsule is a useful device for oral dosing of granular/powdered materials or small amounts of liquid materials and is often used in animal experiments especially in dogs. A gelatin is a protein produced mainly from animal's collagen. The protein digestibility-corrected amino acid score of gelatin is reported to be null due to the absence of tryptophan which is an indispensable amino acid (Schaafsma, 2005; Linder et al., 1997; United States Department of Agriculture (USDA) National Nutrient Database for Standard Reference Release 28, 2015). Therefore, a gelatin is generally considered to be of low nutritional quality. In contrast, some of constituent amino acids for the gelatin are reported to have bioactive effects in practice. Some amino acids including alanine containing into a gelatin are well known to stimulate insulin or glucagon secretion from the pancreas (Rocha et al., 1972; Torres et al., 2009). Insulin and glu- cagon are strongly associated with regulation of glucose homeostasis in the body. However, it is not well known if orally administered gelatin capsules affect these hormone levels in dogs.

In the present study, we investigated the effects of oral administration of gelatin capsules on plasma insulin and glucagon levels in dogs. In addition, plasma alanine level as an index of amino acid absorption from the intestine and some biochemical parameters (glucose, nonesterified fatty acids (NEFA) and total ketone bodies (KB, acetoacetic acid and 3-hydroxybutyric acid)) were also investigated.

\section{MATERIALS AND METHODS}

\section{Animals}

Three male beagle dogs (11 to 13 months old, body weights; 10.7 to $12.9 \mathrm{~kg}$, purchased from Covance Research Products Inc. (Carnegie Center, Princeton, NJ, 
USA)) were used in this study. On the dosing day, the animals were individually housed with a chew toy in an air-conditioned animal room with a 12-hr light-dark cycle (lighting from 7:00 a.m. to 7:00 p.m.) at a temperature of $23 \pm 1^{\circ} \mathrm{C}$, a relative humidity of $55 \pm 5 \%$ and a ventilation rate of 10 to 20 times per hour at Japan Health Sciences Foundation-accredited Toxicology Research Laboratories, Central Pharmaceutical Research Institute, JAPAN TOBACCO INC. The dogs were given a commercial diet (250 g/day, TC-1, Oriental Yeast Co., Ltd., Tokyo, Japan). Tap water was freely available for drinking ad libitum. All animal experimental procedures were approved by the institutional animal care and use committee of the Toxicology Research Laboratories, Central Pharmaceutical Research Institute, JAPAN TOBACCO INC. This study was conducted in accordance with the Japanese Law for the Humane Treatment and Management of Animals (Law No. 105, as revised in 2013, issued in October 1, 1973).

\section{Experimental procedures}

After overnight fasting, each animal was administered orally gelatin capsules (1/2 ounces in size, Torpac Inc., Fairfield, CA, USA). The total amount of gelatin capsules administered was equivalent to the maximum possible oral dosing volume $(15 \mathrm{~mL} / \mathrm{kg}$ ) in dogs (Diehl et al., 2001) and the dose level of gelatin was 0.29 or $1.67 \mathrm{~g} / \mathrm{kg}$, which corresponds to 3 or 17 gelatin capsules per animal, respectively. A small amount of drinking water was given to the dogs immediately after dosing gelatin capsules to make swallowing easier. Food was given after the blood sampling at $4 \mathrm{hr}$ after dosing. For the control data, drinking water was administered orally at a dose volume of $15 \mathrm{~mL} / \mathrm{kg}$ to the same dogs using a catheter on a different day.

\section{Blood sampling}

Blood samples were collected from the forelimb vein without anesthesia once before dosing (as " $0 \mathrm{hr}$ ") and at $0.25,0.5,1,2$ and $4 \mathrm{hr}$ after dosing. An aliquot of each blood sample was transferred into a tube containing sodium heparin and a tube containing aprotinin and EDTA $3 \mathrm{~K}$ and centrifuged $\left(3,000 \mathrm{~min}^{-1}, 4^{\circ} \mathrm{C}, 30 \mathrm{~min}\right)$ to obtain plasma. The heparin-treated plasma samples were used for measurements of insulin, glucose, NEFA, total KB and alanine. Aprotinin and EDTA-3K-treated plasma samples were used for the measurement of glucagon.

\section{Bioanalytical methods}

Plasma insulin and glucagon concentrations were measured using a commercial enzyme-linked immuno- sorbent assay (ELISA) Kit (Morinaga insulin ELISA Kit with a canine insulin standard (Morinaga Institute of Biological Science, Inc., Yokohama, Japan) and YK090 glucagon EIA Kit (Yanaihara Institute Inc., Awakura, Japan)). Plasma glucose, NEFA and total KB concentrations were measured with a TBA-120FR automated analyzer (Toshiba corporation, Tokyo, Japan) using standard reagents by the Hexokinase-G-6-PDH method (Wako Pure Chemical, Tokyo, Japan), the ACS-ACOD Method (Wako Pure Chemical, Tokyo, Japan) and enzyme method (Sanwa Kagaku Kenkyusho Co., Ltd., Nagoya, Japan). Plasma alanine concentrations were determined using a liquid chromatography-tandem mass spectrometry by measuring the alanine derivative which was derivatized by AccQ Fluor reagent kit (Nihon Waters K.K., Tokyo, Japan) after deproteination. Alanine d3 (Cambridge Isotope Laboratories, Inc., Andover, MA, USA) was used as an internal standard.

\section{Statistical analysis}

For plasma insulin, glucagon, glucose, NEFA, total KB and alanine concentrations, delta values between the predosing values $(0 \mathrm{hr})$ and the post-dosing values at each sampling point ( 0.25 to $4 \mathrm{hr}$ ) were calculated. When the values of plasma insulin concentrations were lower than lower limit of qualification $(156 \mathrm{pg} / \mathrm{mL})$, the values were regarded as zero in the calculation. All the data are represented as means and standard deviations (S.D.) for the three animals for each time point. A Dunnett test was used to compare individual data (delta values) after dosing gelatin capsules with those after dosing water (control data) for each sampling point. Simple correlation analyses between plasma insulin or glucagon concentrations and plasma alanine concentrations were conducted using the individual delta data from 0.25 to $4 \mathrm{hr}$ after dosing water or gelatin capsules for each parameter. The levels of significance were set at 5\% and $1 \%$ (two-tailed).

\section{RESULTS}

\section{Plasma insulin and glucagon concentrations}

After dosing water, plasma insulin or glucagon levels did not change for up to $4 \mathrm{hr}$ after dosing (Fig. 1A and 1B). After dosing gelatin capsules, plasma insulin levels rapidly increased with a peak at $0.25 \mathrm{hr}$ at $1.67 \mathrm{~g} / \mathrm{kg}$ when compared with the control values $(\mathrm{p}<0.05)$ and returned to the control levels by $4 \mathrm{hr}$ after dosing (Fig. 1A). Plasma insulin levels tended to increase at $0.29 \mathrm{~g} / \mathrm{kg}$. Plasma glucagon levels also rapidly increased from $0.25 \mathrm{hr}$ after dosing gelatin capsules with a peak at $1 \mathrm{hr}$ after dosing at $1.67 \mathrm{~g} / \mathrm{kg}(\mathrm{p}<0.05)$ and 
Effects of gelatin capsules in dogs

A Insulin

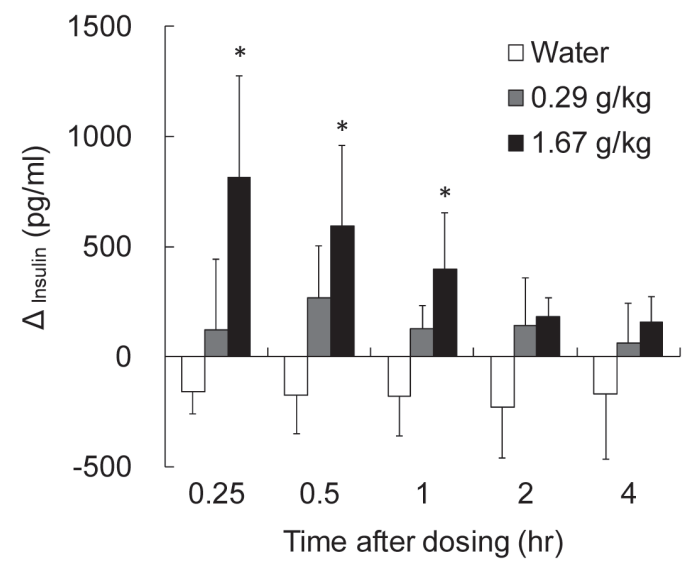

C Glucose

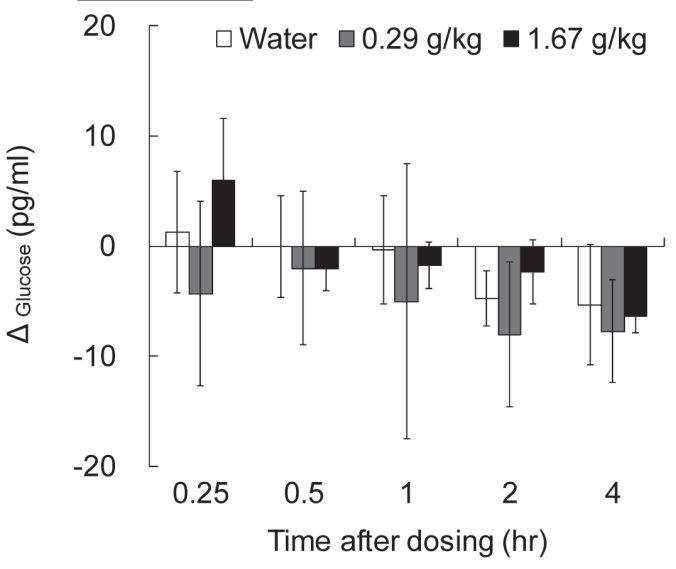

E Total KB

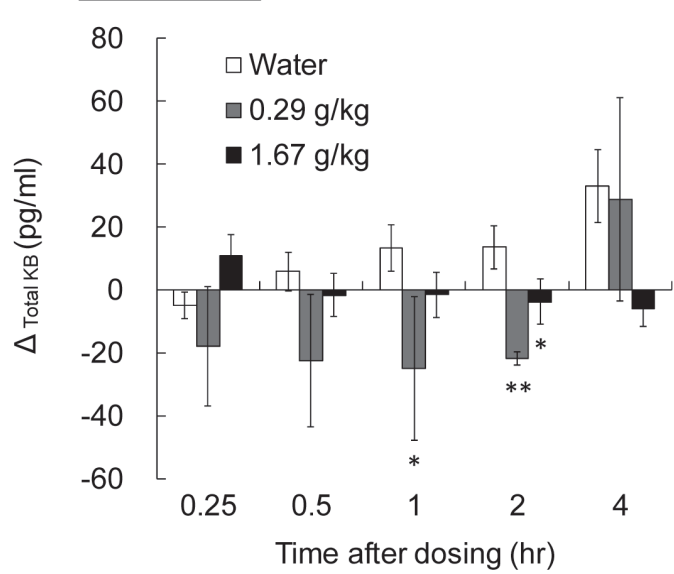

B Glucagon

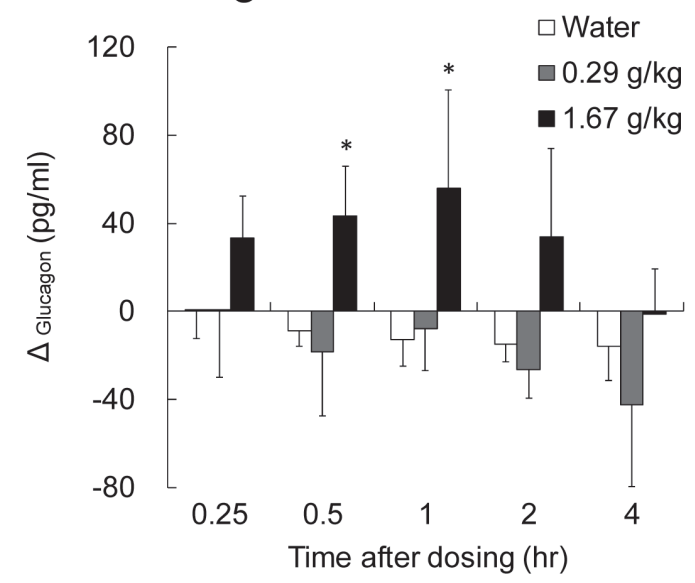

D NEFA

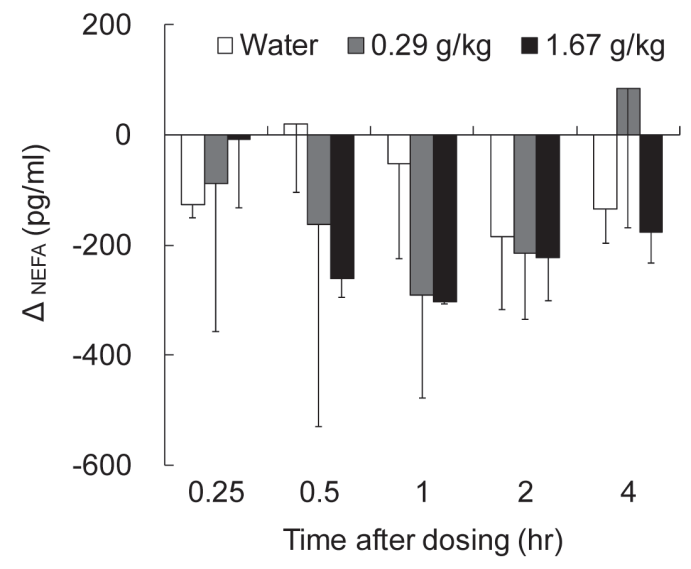

F $\underline{\text { Alanine }}$

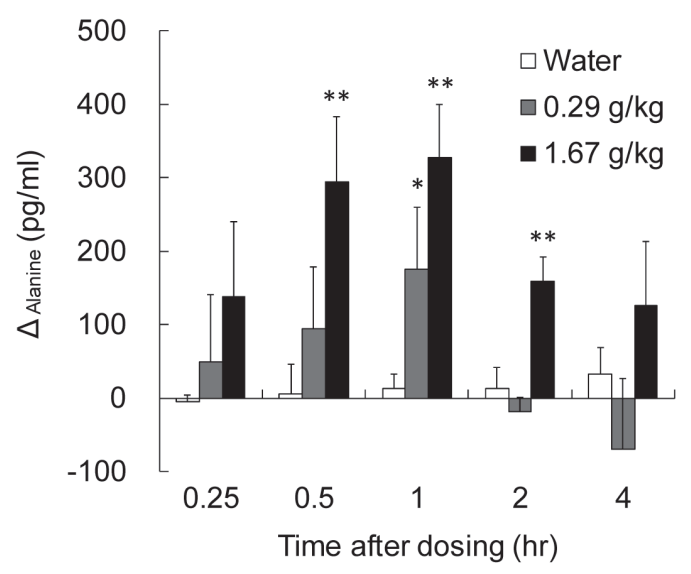

Fig. 1. Changes in plasma insulin, glucagon, glucose, non-esterified fatty acid (NEFA), total ketone body (KB) and alanine concentrations. A: Insulin, B: Glucagon, C: Glucose, D: NEFA, E: total KB, F: Alanine. Data are shown as delta data between the pre-dosing value and the post-dosing value (from 0.25 to $4 \mathrm{hr}$ after dosing) and shown as the mean \pm S.D. of the three animals. ${ }^{*} \mathrm{p}<0.05,{ }^{*} \mathrm{p}<0.01$ : significantly different from water-treatment control experiment (Dunnett test). 
tended to return to the control levels by $4 \mathrm{hr}$ after dosing (Fig. 1B), indicating that the peak of the response of glucagon was later than that of insulin. Plasma glucagon levels did not change at $0.29 \mathrm{~g} / \mathrm{kg}$ unlike insulin. Although the individual baseline values for insulin before gelatin treatment tended to be lower than those before water treatment (Table 1), there were considered to be no biologically meaningful differences.

\section{Plasma insulin-related biochemical parameters}

Plasma glucose levels did not change for up to $4 \mathrm{hr}$ after dosing water or gelatin capsules (Fig. 1C). Plasma NEFA levels also did not change remarkably for up to $4 \mathrm{hr}$ after dosing water or gelatin capsules (Fig. 1D). However, the plasma NEFA levels tended to decrease from 0.5 and $1 \mathrm{hr}$ after dosing gelatin capsules at 0.29 and $1.67 \mathrm{~g} / \mathrm{kg}$ when compared with the control values. Plasma total KB levels gradually increased for up to $4 \mathrm{hr}$ after dosing water (Fig. 1E). After dosing gelatin capsules, plasma total KB levels were stable or decreased for up to $4 \mathrm{hr}$ after dosing at 0.29 and $1.67 \mathrm{~g} / \mathrm{kg}$ and were lower than those at 1 or $2 \mathrm{hr}$ after dosing when compared with the control ( $\mathrm{p}<0.01$ or 0.05$)$ (Fig. 1E).

\section{Plasma alanine concentrations}

Plasma alanine levels did not change for up to $4 \mathrm{hr}$ after dosing water (Fig. 1F). After dosing gelatin capsules, plasma alanine levels increased dose-dependently from $0.25 \mathrm{hr}$ after dosing at 0.29 and $1.67 \mathrm{~g} / \mathrm{kg}$ with a peak at $1 \mathrm{hr}$ when compared with the control values $(\mathrm{p}<0.01$ or 0.05$)$ and returned to the control levels by $4 \mathrm{hr}$ after dosing (Fig. 1F). Correlation analyses using the data after dosing water or gelatin capsules revealed that the delta increase in plasma alanine levels for up to $4 \mathrm{hr}$ after dosing correlated with those in plasma insulin and glucagon levels (Fig. 2, $r^{2}=0.2566(\mathrm{p}<0.01)$ and 0.5213 $(\mathrm{p}<0.01)$ for insulin and glucagon, respectively).

\section{DISCUSSION}

In this study, the effects of orally dosing gelatin capsules on plasma insulin, glucagon and insulin-related biochemical parameters were investigated in dogs following single oral administration of gelatin capsules. The responses in these parameters were monitored for up to $4 \mathrm{hr}$ after dosing. In the results, transient increases in plasma insulin, glucagon and alanine concentrations were observed after dosing gelatin capsules. Furthermore, the increases in plasma insulin and glucagon levels well-correlated with the increase in plasma alanine levels as an index of amino acid absorption from the intestine.

Gelatin is composed of many amino acids except for tryptophan and has high levels of alanine, glycine, proline, glutamic acid, arginine and aspartic acid. These 6 amino acids are reported to account for approximately $60 \%$ of the gelatin by weight (Linder et al., 1997; USDA National Nutrient Database for Standard Reference Release 26). There are two possible mechanisms for the increase in plasma alanine levels after dosing gelatin capsules. One is an increase due to the intestinal absorption of alanine administered orally and the other is an increase due to catabolism of the proteins. After oral dosing gelatin capsules, gelatin was considered to be digested to free amino acids including alanine by several digestive enzymes in the gastro-intestinal lumen followed by intestinal absorption of the free amino acids. On the other hand, since insulin has a strong anabolic action, depletion of insulin is considered to lead to enhancement of protein catabolism in skeletal muscle (Fryburg et al., 1995). In this study, plasma insulin levels rapidly increased after dosing gelatin capsules, indicating that the increased plasma alanine levels is mainly due to intestinal absorption of alanine derived from the orally-administered gelatin capsules. Similarly, the amino acids composing gelatin capsules were considered to be absorbed from

Table 1. Individual baseline data for each parameter.

\begin{tabular}{|c|c|c|c|c|c|c|c|c|c|}
\hline \multirow{2}{*}{$\begin{array}{l}\text { Animal No.: } \\
\text { Treatment: }\end{array}$} & \multicolumn{3}{|c|}{1} & \multicolumn{3}{|c|}{2} & \multicolumn{3}{|c|}{3} \\
\hline & W & $\mathrm{G}(0.29)$ & $\mathrm{G}(1.67)$ & W & $\mathrm{G}(0.29)$ & $\mathrm{G}(1.67)$ & $\mathrm{W}$ & $\mathrm{G}(0.29)$ & $\mathrm{G}(1.67)$ \\
\hline (Parameter) & & & & & & & & & \\
\hline Insulin (pg/mL) & 502 & 250 & 123 & 399 & $<156$ & $<156$ & 254 & 345 & 190 \\
\hline Glucagon (pg/mL) & 146 & 161 & 160 & 314 & 294 & 204 & 93 & 96 & 118 \\
\hline Glucose (mg/dL) & 107 & 115 & 100 & 96 & 102 & 102 & 102 & 101 & 98 \\
\hline $\operatorname{NEFA}(\mathrm{mg} / \mathrm{dL})$ & 568 & 322 & 433 & 896 & 740 & 482 & 1021 & 1177 & 540 \\
\hline Total KB $(\mu \mathrm{mol} / \mathrm{L})$ & 30 & 42 & 34 & 47 & 47 & 37 & 41 & 84 & 17 \\
\hline Alanine $(\mathrm{pg} / \mathrm{mL})$ & 324 & 573 & 390 & 243 & 305 & 245 & 249 & 438 & 209 \\
\hline
\end{tabular}

W: Baseline data from water-treatment control experiment, G (0.29): Baseline data from gelatin-treated experiment at $0.29 \mathrm{~g} / \mathrm{kg}$, $\mathrm{G}$ (1.67): Baseline data from gelatin-treated experiment at $1.67 \mathrm{~g} / \mathrm{kg}$, NEFA: Non-esterified fatty acids, KB: Ketone bodies, Lower limit of qualification for insulin was $156 \mathrm{pg} / \mathrm{mL}$. 
Effects of gelatin capsules in dogs

A

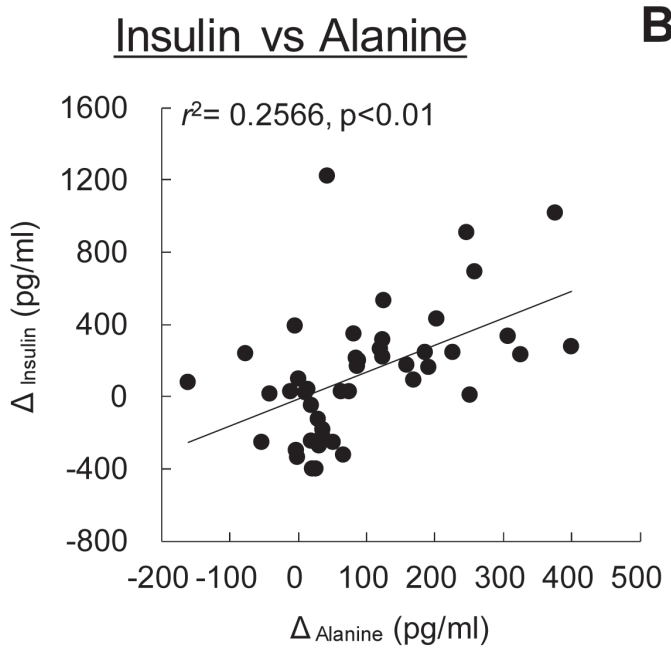

B

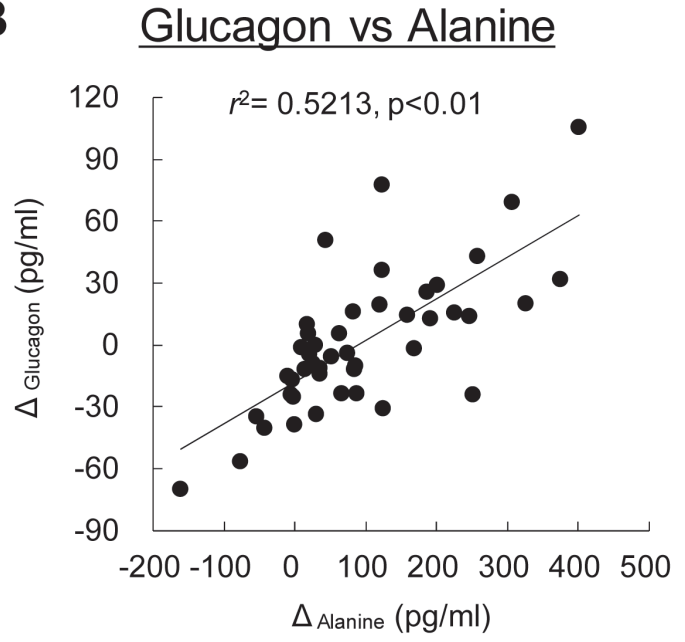

Fig. 2. Correlation between plasma insulin or glucagon concentrations and plasma alanine concentration. A: Insulin vs Alanine, B: Glucagon vs Alanine. Correlation analyses were conducted using the individual delta data between the pre-dosing values and the post-dosing values of plasma insulin, glucagon and alanine concentrations obtained after dosing water or gelatin capsules.

the intestine.

Insulin and glucagon secretions are reported to be stimulated by many amino acids including alanine, glycine, proline, glutamic acid, arginine and aspartic acid as reported in an in vivo study using dogs treated with intravenous injection of each amino acid at $1 \mathrm{mmol} / \mathrm{kg}$ (Rocha et al., 1972). The mechanisms by which several amino acids enhance insulin secretion are known to be varied and depend on each amino acid (Torres et al., 2009). Alanine is co-transported with $\mathrm{Na}^{+}$into the $\beta$-cells from plasma and induces insulin secretion in association with elevation of the intercellular $\mathrm{Na}^{+}$(Dunne et al., 1990). Arginine activates voltage-gated $\mathrm{Ca}^{2+}$ channels and increases exocytosis of insulin vesicles in the $\beta$-cells by direct depolarization of the plasma membrane at neutral pH (Blachier et al., 1989). Alanine, glycine and glutamine are also reported to be associated with the stimulation of incretin release from the intestinal L-cells leading to stimulation of insulin secretion (Gameiro et al., 2005; Reimann et al., 2004). On the other hand, the mechanisms by which amino acids enhance glucagon secretion are unclear (Rorsman et al., 2008). Glucagon is a peptide hormone secreted from the pancreatic $\alpha$-cells and is recognized as a counter hormone against insulin having a role in glucose catabolism (Quesada et al., 2008). In this study, the peak of the increase in plasma glucagon levels was a little later than that in plasma insulin levels after dosing gelatin capsules. Therefore, the elevation of plasma glucagon levels was considered to be due to not only a direct action of the amino acids on glucagon secretion but also a compensatory increase in glucagon secretion to the anabolic action of insulin.

Plasma glucose levels did not change although plasma insulin levels rapidly increased after dosing gelatin capsules in this study. The absence of alteration of the plasma glucose levels was considered to be due to strictly controlled glucose homeostasis in association with the rapid compensatory secretion of glucagon.

Plasma NEFA levels tended to decrease after the oral dosing of gelatin capsules. The decrease in plasma NEFA levels was likely due to the inhibitory effects of insulin on lipolysis. Although plasma total KB levels gradually increased for up to $4 \mathrm{hr}$ after dosing water, these were considered to be due to the enhancement of $\beta$-oxidation in response to prolonged fasting (Hashimoto et al., 2000). In contrast, plasma total $\mathrm{KB}$ levels did not change or decreased after dosing gelatin capsules during test period. This seemed to be related to the decrease in plasma NEFA levels because KB is derived from acetyl-CoA produced by $\beta$-oxidation of NEFA.

In conclusion, oral administration of the gelatin capsules induced slight but significant effects based on the systemic insulin and glucagon actions through intestinal absorption of amino acids. These effects were transient even after the dosing of a large amount of gelatin capsules and were likely minimized by metabolic compensation of insulin and glucagon. However, this study showed that insulin and glucagon secretions related to dosing gel- 
H. Yokoyama et al.

atin capsules could lead to modify understanding of the pharmacological or toxicological action of hypoglycemic drugs or drugs affecting lipid metabolism. Therefore, attention should be paid to the effect of gelatin on the insulin and glucagon secretions at conducting animal experiments using a gelatin capsule.

\section{ACKNOWLEDGMENTS}

The authors would like to thank the invaluable contributions of the staff at the Toxicology Research Laboratories, Central Pharmaceutical Research Institute, JAPAN TOBACCO INC.

Conflict of interest---- The authors declare that there is no conflict of interest.

\section{REFFERENCES}

Blachier, F., Mourtada, A., Sener, A. and Malaisse, W.J. (1989): Stimulus-secretion coupling of arginine-induced insulin release. Uptake of metabolized and nonmetabolized cationic amino acids by pancreatic islets. Endocrinology, 124, 134-141.

Diehl, K.H., Hull, R., Morton, D., Pfister, R., Rabemampianina, Y., Smith, D. and Vorstenbosch, C.V.D. (2001): A Good Practice Guide to the Administration of Substances and Removal of Blood, Including Routes and Volumes. J. Appl. Toxicol., 21, 15-23.

Dunne, M.J., Yule, D.I., Gallacher, D.V. and Petersen, O.H. (1990): Effects of alanine on insulin-secreting cells: Patch-clamp and single cell intracellular $\mathrm{Ca}^{2+}$ measurements. Biochim. Biophys. Acta, 1055, 157-164.

Fryburg, D.A., Jahn, L.A., Hill, S.A., Oliveras, D.M. and Barrett, E.J. (1995): Insulin and insulin-like growth factor-1 enhance human skeletal muscle protein anabolism during hyperami- noacidemia by different mechanisms. J. Clin. Invest., 96, 17221729.

Gameiro, A., Reimann, F., Habib, A.M., O’Malley, D., Williams, L., Simpson, A.K. and Gribble, F.M. (2005): The neurotransmitters glycine and GABA stimulate glucagon-like peptide-1 release from the GLUTag cell line. J. Physiol., 569, 761-772.

Hashimoto, T, Cook, W.S., Qi, C., Yeldandi, A.V., Reddy, J.K. and Rao, M.S. (2000): Defect in peroxisome proliferator-activated receptor a-inducible fatty acid oxidation determines the severity of hepatic steatosis in response to fasting. J. Biol. Chem., 275, 28918-28928.

Linder, M., Rozan, P., Kossori, R.L., Fanni, J., Villaume, C., Mejean, L. and Parmentier, M. (1997): Nutritional value of veal bone hydrolysate. J. Food Sci., 62, 183-189.

Quesada, I., Tuduri, E., Ripoll, C. and Nadal, A. (2008): Physiology of the pancreatic $\alpha$-cells and glucagon secretion: role in glucose homeostasis and diabetes. J. Endocrinol., 199, 5-19.

Reimann, F., Williams, L., da Silva Xavier, G., Rutter, G.A. and Gribble, F.M. (2004): Glutamine potently stimulates glucagonlike peptide-1 secretion from GLUTag cells. Diabetologia, 47, 1592-1601.

Rocha, D.M., Faloona, G.R. and Unger, R.H. (1972): Glucagonstimulating activity of 20 amino acids in dogs. J. Clin. Invest., 51, 2346-2351.

Rorsman, P., Salehi, S.A., Abdulkader, F., Braun, M. and MacDonald, P.E. (2008): $\mathrm{K}_{\mathrm{ATP}}$-channels and glucose-regulated glucagon secretion. Trends Endocrinol. Metab., 19, 277-284.

Schaafsma, G. (2005): The Protein Digestibility-Corrected Amino Acid Score (PDCAAS)--a concept for describing protein quality in foods and food ingredients: a critical review. J. AOAC Int., $\mathbf{8 8}, 988-994$

Torres, N., Noriega, L. and Tovar, A.R. (2009): Nutrient modulation of insulin secretion. Vitam. Horm., 80, 217-244.

United States Department of Agriculture (USDA) (2015): Full Report (All Nutrients) 19177, Gelatins, dry powder, unsweetened. USDA National Nutrient Database for Standard Reference Release 28. Available: https://ndb.nal.usda.gov/ndb/foods/show/ 6205? format $=$ Full\&reportfmt $=$ pdf\&pdfQvs $=\% 7 \mathrm{Bn} 1 \% 3 \mathrm{D} \% 7 \mathrm{BQ}$ $\mathrm{v} \% 3 \mathrm{D} 1 \% 7 \mathrm{D} \% 2 \mathrm{C}+\mathrm{Qv} \% 3 \mathrm{D} 1 \% 7 \mathrm{D} \& \mathrm{ds}=$ [22 January 2017] 